Document downloaded from:

http://hdl.handle.net/10251/120636

This paper must be cited as:

Sánchez-Marín, JR.; Bachiller Martin, MC.; Juliá Morte, M.; Nova-Giménez, V.; Esteban González, H.; Boria Esbert, VE. (2018). Microwave Filter based on Substrate Integrated Waveguide with Alternating Dielectric Line Sections. IEEE Microwave and Wireless Components Letters. 28(11):990-992. https://doi.org/10.1109/LMWC.2018.2871644

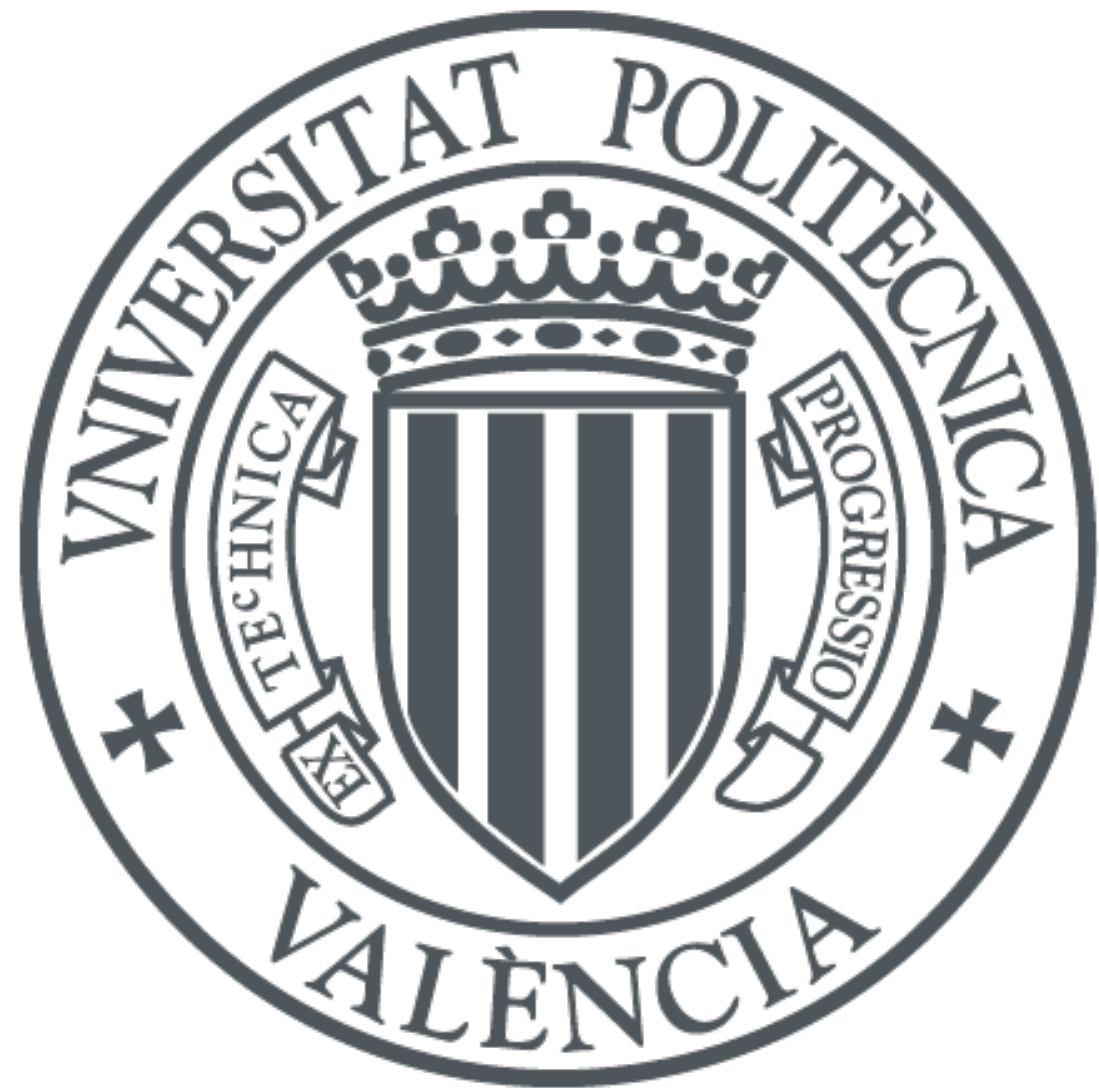

The final publication is available at

http://doi.org/10.1109/LMWC.2018.2871644

Copyright Institute of Electrical and Electronics Engineers

Additional Information

(c) 2018 IEEE. Personal use of this material is permitted. Permission from IEEE must be obtained for all other users, including reprinting/ republishing this 


\title{
Microwave Filter based on Substrate Integrated Waveguide with Alternating Dielectric Line Sections
}

\author{
Juan R. Sánchez, Carmen Bachiller, María Juliá, Vicente Nova, Héctor Esteban and Vicente E. Boria \\ Instituto de Telecomunicaciones y Aplicaciones Multimedia. Universitat Politècnica de València. \\ Camino de Vera, s/n, 46021 Valencia, Spain. \\ e-mail: juasncm1@upv.es,mabacmar@dcom.upv.es
}

\begin{abstract}
A new topology of microwave filter based on Substrate Integrated Waveguide (SIW) with alternating dielectric line sections is presented in this paper. The two alternating sections are waveguides without and with dielectric material acting, respectively, as the inverters and the resonant sections of the proposed filter. This topology allows to easily design and manufacture compact filters with lower losses and higher rejection band than other available SIW realizations. For validation purposes, a prototype of a 4-pole bandpass filter, based on alternating dielectric line sections, has been designed and manufactured, including successful experimental results.
\end{abstract}

Index Terms-Substrate Integrated Waveguide (SIW), planar structure, filter, alternating dielectric line sections.

\section{INTRODUCTION}

$\mathbf{M}$ ICROWAVE filters are key components in high frequency communication systems, whose requirements are recently increased. Among them, the need for low cost devices with reduced mass and volume, and their potential integration with the current planar technology are highlighted. Substrate Integrated Waveguide (SIW) technology appeared to solve these problems [1]. It integrates a rectangular waveguide into a planar substrate, obtaining devices that are more compact, and can be easily manufactured with cheaper techniques.

SIW technology has been successfully employed to implement different bandpass filter topologies. Among them, a widely used solution is based on direct-coupled cavity resonators [2]. An alternative SIW filtering structure has been recently proposed in [3], based on the periodic perforation of the dielectric substrate with air holes in a constant width structure. Being a very promising solution, it has some fabrication issues related to the requested number (and dimensions) of perforated holes.

The width of the stopband rejection is another important requirement for next generation SIW-based filters. Several techniques have been developed to enhance the stopband behaviour of these filters (see [4]), but they still have some limitations in terms of the achieved stopband frequency range.

In this paper, a new type of bandpass filter, with enhanced behaviour in terms of the previous limitations, is proposed. Its topology is based on alternating line sections, without and with dielectric material. This filter topology allows to reduce

This work was partially funded by the Generalitat Valenciana research project PROMETEOII/2015/005, by the Ministerio de Educación, Cultura y Deporte (Spain) under the Fellowship Program for Training University Professors FPU14/00150, and by Ministerio de Economía y Competitividad (Spain) under R\&D project TEC2016-75934-C4-1-R. the related losses and to enhance the stopband behaviour. A 4-pole bandpass prototype was designed, manufactured and compared with alternative SIW realizations.

\section{Filter Topology AND DESIGN}

The layout of the proposed filtering device is a succession of substrate integrated line sections with and without dielectric (see central layer in Fig. 1). In the empty line sections, the field is confined laterally using metallized walls. And in the dielectric line sections, metallized via holes, as in the traditional SIW, are used. A metallic top and bottom layer are finally added for confining the field in the empty line sections vertically. For a filter of order $N$, with $N$ reflection zeros, $N$ line sections with dielectric of lengths $l_{\text {Res }}^{(i)}(i \in 1 \cdots N)$ are used, acting as resonators. The empty line sections, where the fundamental mode is below cut-off, act as inverters, implementing the coupling between resonators and with the input and output lines. This gives $N+1$ empty lines sections of length $l_{W i n}^{(i)}(i \in 1 \cdots N+1)$.

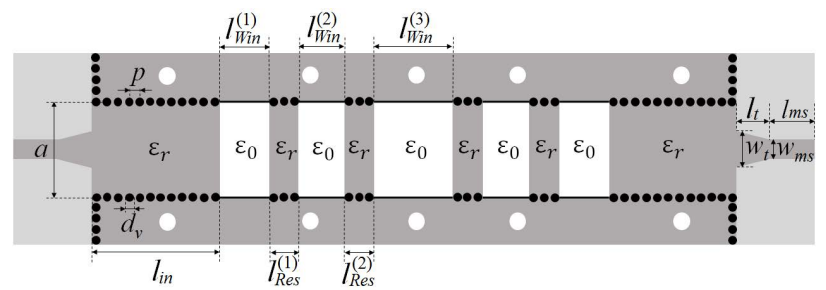

Fig. 1. Layout of the central substrate of the filter based on alternating dielectric line sections (with $N=4$ ). In black the metallized vias and the border copper metallization, in dark gray the copper metallization on top of the layer, in light gray the dielectric substrate, and in white the air-filled sections and the fastening screw holes. To close the air-filled sections, top and botton copper covers are used (not shown here).

In order to test the validity of the proposed structure as a filter, and to establish a consistent design procedure, a simple filter with only one resonator is first designed using the standard procedure of [5], which uses an equivalent ideal network composed of ideal inverters and resonators as a reference. In Fig. 2 we show the equivalent network for this 1-pole filter, designed for providing a Chebyshev response centred at 11 $\mathrm{GHz}$, with $300 \mathrm{MHz}$ bandwidth and return losses of $10 \mathrm{~dB}$. The input and output lines of the filter will be SIW lines filled with dielectric of $\varepsilon_{r}=6$ (Rogers TMM 6 substrate of height $h=1.27 \mathrm{~mm}$ ), with vias of diameter $d_{v}=0.8 \mathrm{~mm}$, and a separation between vias of $p=1 \mathrm{~mm}$. The equivalent width of 
the SIW is $a=8 \mathrm{~mm}$. According to [5], the desired response is achieved with the ideal equivalent network when the inverters have normalized inversion constants $\bar{K}_{S, 1}=\bar{K}_{1, L}=0.3529$.

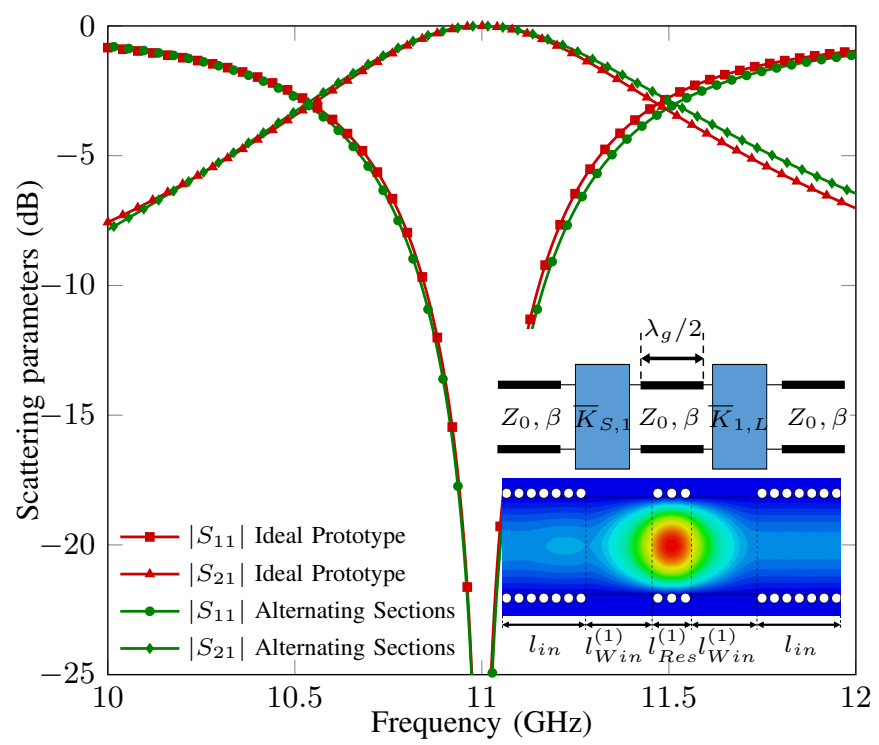

Fig. 2. Results for 1-pole basic filter. The input and output feeding SIW lines and the dielectric-filled sections are identified by the rows of vias, the air-filled sections have solid lines.

Considering the topology proposed in this work (as shown in Fig. 1 but with $N=1$ ), the ideal inverter of our simple filter will be implemented with an empty line section of length $l_{\text {Win }}^{(1)}$. So, an empty line section below cutoff of width $a=8 \mathrm{~mm}$, accessed with SIW lines, is simulated (using the fast mode-matching method of [6], which considers the SIW line sections as closed rectangular waveguides filled with dielectric). The length $l_{\text {Win }}^{(1)}$ is optimized until the amplitude of the refection coefficient is the same than that of the ideal inverter, which happens when $l_{\text {Win }}^{(1)}=3.204 \mathrm{~mm}$. The next step is to find out where the reference planes have to be placed along the SIW accessing lines in order to have the same phase of the reflection coefficient than that of an ideal inverter (i.e. phase $\pi$ ). This happens when $l_{\text {corr }}^{(1)}=-2.1661 \mathrm{~mm}$. So, the empty line section together with two accessing SIW lines filled with dielectric of lengths $l_{\text {corr }}^{(1)}$ behaves as an ideal inverter. When both adjacent inverters are connected to the resonator (a SIW line of length $\lambda_{g_{0}} / 2$ ), the total length of the SIW line between the two empty sections is $l_{\text {Res }}^{(1)}=\lambda_{g_{0}} / 2+2 \cdot l_{\text {corr }}^{(1)}=7.7414-2 \cdot 2.1661=3.4092 \mathrm{~mm}$. These values of $l_{W i n}^{(1)}$ and $l_{\text {Res }}^{(1)}$ are used as an initial point, and are fine optimized with the mode-matching method of [6]. The resulting values are then used as an initial point for a new optimization with a commercial electromagnetic software (CST), which simulates the SIW lines with rows of metallized vias, and adjusts the response to that of the ideal network as much as possible. Fig. 2 shows the reflection and transmission coefficients of the single resonator filter with the values of $l_{W i n}^{(1)}$ and $l_{R e s}^{(1)}$ obtained with this procedure. These parameters are compared with those of the ideal network, and a very good agreement can be observed. The electric field distribution inside the single resonator filter is also included in Fig. 2. It can be observed that the electromagnetic field is concentrated in the line sections filled with dielectric, which validates the idea that it behaves as a resonator, and that its short length is consequence of the negative lengths that have been added in order to implement the ideal inverter with the empty line section. The field distribution also proves that the empty line sections behave as the filter coupling elements. This structure can be repeated for filters with any arbitrary number of resonators $(N)$.

\section{RESULTS}

Following this method, a 4-pole filter with a Chebysheb bandpass response, centered at $11 \mathrm{GHz}$ with $0.01 \mathrm{~dB}$ ripple in a $300 \mathrm{MHz}$ bandwidth, has been designed (considering the same Rogers TMM6 substrate, and a copper cladding of 17.5 um). Moreover, the tapered transition from mcirostrip to SIW has been synthesized as proposed in [7]. The dimensions of the filter and the transition, as depicted in Fig. 1, are shown in Table I.

TABLE I

DIMENSIONS (IN MM) OF THE FILTER AND TAPERED TRANSITION.

\begin{tabular}{ll|ll|ll}
\hline$a$ & 8.000 & $l_{R e s}^{(1)}$ & 3.011 & $p$ & 1.000 \\
$l_{W i n}^{(1)}$ & 3.708 & $l_{\text {Res }}^{(2)}$ & 2.943 & $d_{v}$ & 0.800 \\
$l_{W i n}^{(2)}$ & 8.366 & $w_{t}$ & 3.084 & $w_{m s}$ & 1.800 \\
$l_{W i n}^{(3)}$ & 9.283 & $l_{t}$ & 2.201 & $l_{m s}$ & 5.000
\end{tabular}

A prototype of this 4-pole bandpass filter has been manufactured as shown in Fig. 3. The top and bottom covers are just two metallic layers with alignment holes and fastening screws. The central layer contains the microstrip line that can integrate the filter to any planar substrate, the transitions from microstrip to the SIW input lines and the filter itself.

The fabrication process for producing the body consists on drilling the vias in the filled sections, and cutting the walls in the empty ones. Then, the substrate is metallized, and afterwards the top metallic layer is milled to form the transition and the microstrip line. Finally, the device is cut and separated from the substrate sheet, and all the layers are duly fastened and welded.

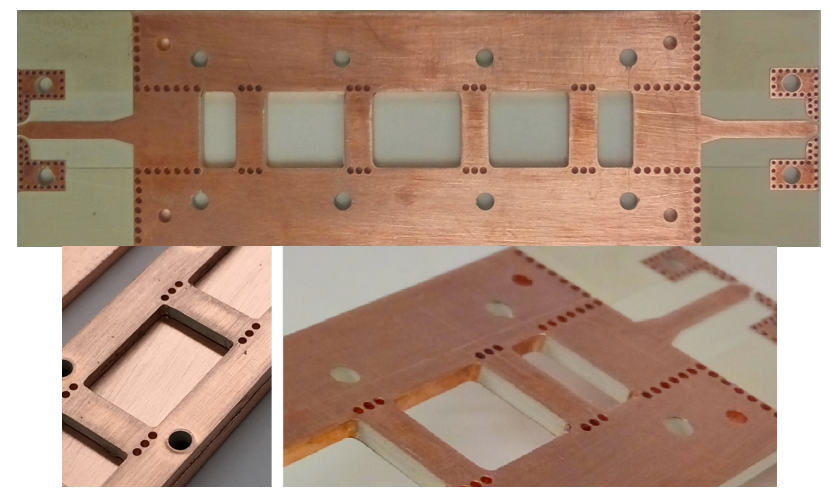

Fig. 3. Manufactured filter. On top, the central layer. Bottom, detailed views (on the left with bottom cover) showing the discontinuities between sections and the side wall conductors. 
In Fig. 4, the measured frequency response of the manufactured prototype is compared with the simulation data obtained with CST. A TRL calibration kit was used to eliminate the effect of connectors and transitions. There is a very good agreement between both results. In order to fairly compare the proposed topology with other available SIW realizations, the parameters of similar filter implementations are shown in Table II. The same filter specifications and substrate are used in all of them: $11 \mathrm{GHz}$ of central frequency, $2.7 \%$ of relative bandwidth, and dielectric substrate TMM6 $\left(\epsilon_{r}=6\right.$, $\tan \delta=0.0023$ ), except for the case of ESIW, which is empty. The $Q$-factor has been estimated applying the formula in [8].

The proposed structure presents several advantages: its associated $Q$-factor is better than for the SIW filter, it is easy to design, it is simple to manufacture, and their inverters can be done more accurately (since the use of vias for implementing narrower SIW widths is avoided). Obviously, ESIW presents higher $Q$-factor due to the absence of dielectric substrate in the whole structure, but the ESIW filter is bigger than the proposed one and requires a more complex transition [9].

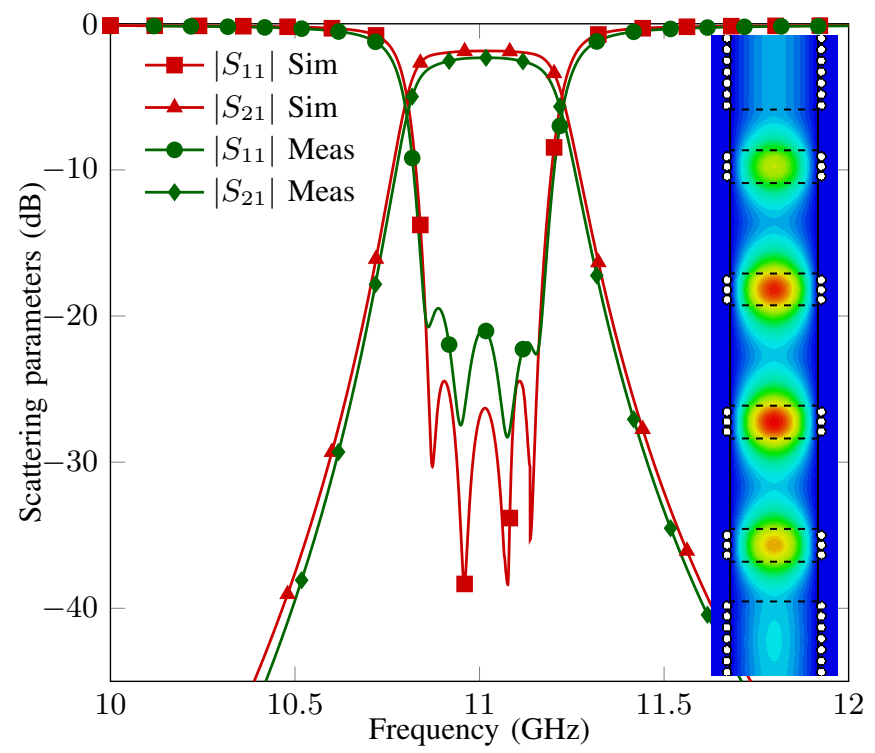

Fig. 4. Comparison between simulated and measured frequency responses for the 4-pole filter. On the right, the E-field distribution for the central frequency.

TABLE II

COMPARISON BETWEEN THE FILTER BASED ON ALTERNATING DIELECTRIC LINE SECTIONS (ADLS) AND SIW FILTERS.

\begin{tabular}{lccc}
\hline & $A D L S$ & $S I W$ & $E S I W$ \\
\hline$Q$-factor & 350 & 273 & 1226 \\
$1^{\text {st }}$ spurious frequency & $2 f_{0}$ & $1.5 f_{0}$ & $1.5 f_{0}$ \\
Width $(\mathrm{mm})$ & 8.65 & 8.65 & 19.05 \\
Length $(\mathrm{mm})$ & 45 & 30 & 77
\end{tabular}

Fig. 5 shows the out-of-band response of the filter prototype. The stopband behaviour (with an attenuation higher than $35 \mathrm{~dB}$ ) can reach values up to $20 \mathrm{GHz}$ (around $2 f_{0}$, instead of $1.5 f_{0}$ as conventional SIW realizations [2]). The cut-off frequency of the spurious band that appears at the second resonance of the resonators, whose mode is $T E_{102}$, depends on the resonator length, permittivity and width. In this particular realization the length of the resonators is very short, thus increasing the cut-off frequency of this spurious band beyond $2 f_{0}$. The spurious band that appears when the air-filled sections begin to concentrate the electrical field and behave as resonators rather than as inverters is also located beyond $2 f_{0}$.

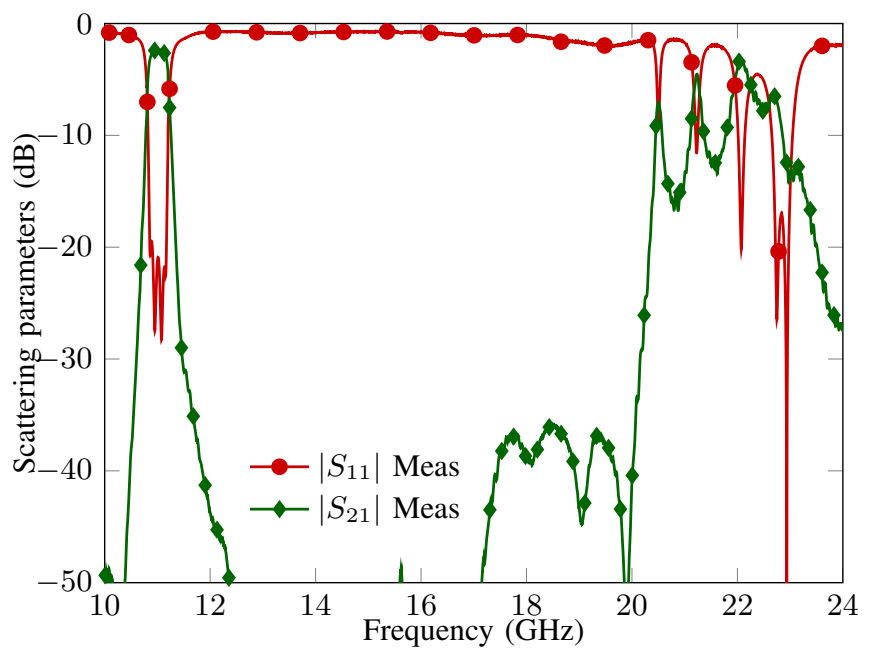

Fig. 5. Out of band behaviour for the filter prototype.

\section{CONCLUSION}

A novel filter topology, based on alternating dielectric line sections, has been proposed and experimentally validated. Standard design procedures for coupled cavities filters can be used to easily design this topology. The new filter topology improves the $Q$-factor when compared to a classical SIW technology, is smaller that the same filter in ESIW, and presents a good out-of-band response.

\section{REFERENCES}

[1] D. Deslandes and K. Wu, "Integrated microstrip and rectangular waveguide in planar form," IEEE Microw. and Wireless Components Letters, vol. 11, no. 2, pp. 68-70, February 2001.

[2] X. P. Chen and K. Wu, "Substrate integrated waveguide filter: Basic design rules and fundamental structure features," IEEE Microwave Magazine, vol. 15, no. 5, pp. 108-116, July 2014.

[3] L. Silvestri, E. Massoni, C. Tomassoni, A. Coves, M. Bozzi, and L. Perregrini, "Substrate integrated waveguide filters based on a dielectric layer with periodic perforations," IEEE Transactions on Microwave Theory and Techniques, vol. PP, no. 99, pp. 1-11, 2017.

[4] X. P. Chen, K. Wu, and D. Drolet, "Substrate integrated waveguide filter with improved stopband performance for satellite ground terminal," IEEE Transactions on Microwave Theory and Techniques, vol. 57, no. 3, pp. 674-683, March 2009.

[5] G. Matthaei, E. Jones, and L. Young, Microwave Filters ImpedanceMatching Networks, and Coupling Structures. Artech House Publishers, February, 1980

[6] J. R. Sanchez, C. Bachiler, M. Juliá, and H. Esteban, "Modal method for the efficient analysis and design of microwave filters based on multiple discontinuities," in 17th Mathematical Modelling Conference in Engineering and Human Behaviour., July 2017, pp. 284-289.

[7] E. Diaz, A. Belenguer, H. Esteban, O. Monerris, and V. E. Boria, "A novel transition from microstrip to a substrate ntegrated waveguide with higher characteristic impedance," in 2013 IEEE MTT-S International Microwave Symposium Digest (MTT), June 2013, pp. 1-4.

[8] I. Hunter, Theory and Design of Microwave Filters. London: The Institution of Electrical Engineers, 2001.

[9] A. Belenguer, H. Esteban, and V. E. Boria, "Novel empty substrate integrated waveguide for high performance microwave integrated circuits," IEEE Trans. Microwave Theory and Techniques, vol. 62, no. 4, pp. 832839, April 2014. 